\title{
Increased Serum Alkaline Phosphatase in a FeLV-Positive Cat
}

\author{
Stella de Faria Valle1, Bárbara Schwarzbach Elesbão², Fernanda Vieira Amorim da Costa², Laís Stefen Gambin ${ }^{1}$, \\ Luciana de Jesus², Mauricio Bianchini Moresco ${ }^{2}$, Marcelle Bettio² \& Félix Hilário Diaz González
}

\begin{abstract}
Background: Feline Leukemia Virus (FeLV) is an oncogenic virus that usually invades bone marrow causing non-regenerative anemia and thrombocytopenia. In FeLV positive cats, the myeloid leukemia is characterized by intense proliferation of blast cells in a bone marrow with myeloid precursor predominance and several malignancies degree. The leukocyte alkaline phosphatase is found secondary granules of mature neutrophils of humans, horses and cows but not in dogs and cats, which demonstrate the enzyme activity in myeloblast and promyeloblasts cells. In this case is describing a FeLV-positive case with leukemic disorder accompanied by extremely high levels of serum alkaline phosphatase.

Case: A 8-year-old castrated male Domestic Short Hair cat with a 3-month history of convulsions, pica, vomits, lethargy, hypodipsia and anorexia was examined and showed mild dehydration, severe jaundice and hyperpnoea. Laboratory results evidenced severe normocytic hypochromic non-regenerative anemia, moderated leukocytosis with severe degenerative neutrophil left shift, moderated lymphocytosis and severe thrombocytopenia. Serum biochemical profile showed mild hyperalbuminemia, moderated increment in alanine transaminase and severe increment in alkaline phosphatese. The cat was positive for ELISA point of care FeLV antigen and negative for FIV antibodies. Urinalysis revealed moderated bilirubinuria and proteinuria. The bone marrow cytology showed high cellularity, increased of M:E ratio, rare megakaryocyte lineage, erythroid hypoplasia, myeloid hyperplasia with blast excess and dysgranulopoiesis.

Discussion: FeLV is a virus that usually causes cytopenias and in this case this was well evidenced through a severe nonregenerative anemia and thrombocytopenia. Generally, anemia is a non-neoplastic complication of FeLV mainly related to suppressive effects hematopoietic and stromal cells of the bone marrow. Nevertheless, in this case, the anemia also may be attributable to the observed acute myeloid leukemia. Neutrophilia is an uncommon finding in FeLV-infected cats, even more with the severe degenerative left shift as in the present case. This finding could be associated to a myeloid hyperplasia. Some biochemical alterations, as hyperalbuminemia and increment in values of serum ALT could be explained by clinical observations like dehydration and some degree of hepatic enlargement, respectively. However, the excessive value for serum alkaline phosphatase activity is an unpublished finding in cats. In humans high levels of ALP has been associated with neutrophilia and it is quite likely that a great amount of degenerative neutrophils may contribute for high leakage of ALP than in other circumstances of neutrophilia. This is the first report of a FeLV-positive cat exhibiting extremely high serum values of the enzyme ALP, possibly attributable to acute myeloid leukemia.
\end{abstract}

Keywords: neutrophilia, cytopenia, non-regenerative anemia, acute leukemia. 


\section{INTRODUCTION}

Feline leukemia virus (FeLV) is a worldwide distributed virus that has oncogenic properties. The virus usually invades bone marrow leading to non-regenerative anemia and myelodysplastic disorders [1]. Anemia is the most common non-neoplastic complication in a FeLV positive cat [1] and approximately $10 \%$ are regenerative [3]. The frequent non-regenerative anemia in the FeLV infection is related with primary infection in the bone marrow hematopoietic and stromal cell causing a suppressive effect [3]. Neutrophilia is an uncommon finding in FeLV-infected cats [2] and no reports show severe increases of clinical enzymes in those patients. The aim of this study was to present a case of a FeLV-positive cat with uncommon neutrophilia and lymphocytosis accompanied by extremely high levels of serum alkaline phosphatase.

\section{CASE}

A 8-year-old castrated male Domestic Short Hair cat was referred to the Veterinary Hospital of the Federal University of Rio Grande do Sul (Porto Alegre, Brazil) with a 3-month history of convulsions, pica, vomits, lethargy, hypodipsia and anorexia. Physical examination revealed a mild dehydration, severe jaundice and hyperpnoea (40 bpm; RI 10-30). Abnormalities in CBC (Procyte Dx $)^{1}$ included severe normocytic hypochromic non-regenerative anemia (8\% HCT, RI 24-45; $2.4 \mathrm{~g} / \mathrm{dL}$ hemoglobin, RI 8-15 g/dL; 0\% reticulocytes, $\mathrm{RI}<0,4)$, moderated leukocytosis $(27,600 / \mu \mathrm{L}, \mathrm{RI} 5,000-19,500 / \mu \mathrm{L})$ with severe degenerative left shift $(1,656 / \mu \mathrm{L}$ myelocytes, RI 0; 3,036/ $\mu \mathrm{L}$ metamyelocytes, RI 0; 3,864/ $\mu \mathrm{L}$ band neutrophils, $\mathrm{RI}<300 / \mu \mathrm{L})$, moderated lymphocytosis $(12,400 /$

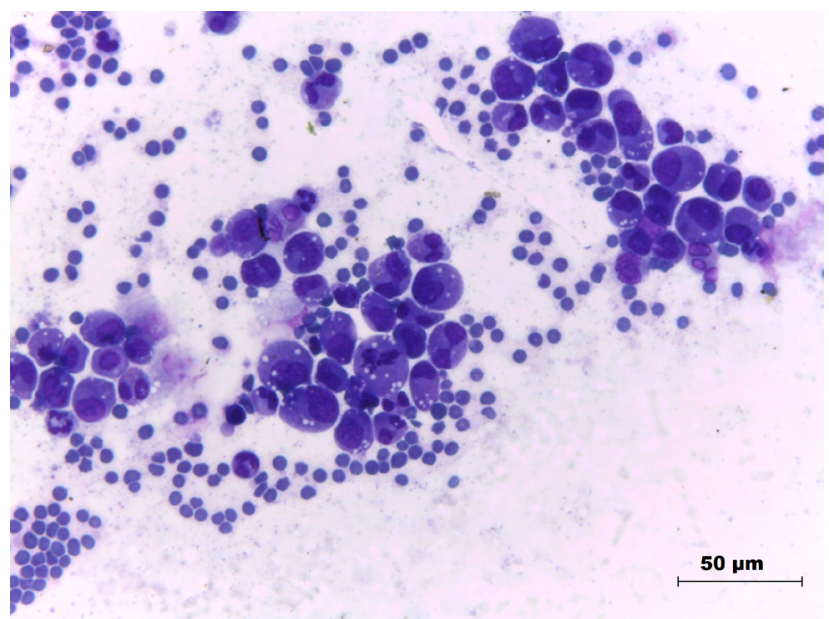

Figure 1. Bone marrow cytology. Increased $M$ :E ratio due to myeloid hyperplasia with blast excess. The dysgranulopoiesis could see visualized in pleomorphic myeloid blast cells with abnormal nuclei conformation, citoplasmatic vacuolization and maturation asynchrony. Abnormal mitotic figure. Wright Stain [magnification: 40x]. $\mu \mathrm{L}, \mathrm{RI} 1,500-7,000 / \mu \mathrm{L})$ and severe thrombocytopenia $(50,000 / \mu \mathrm{L}, \mathrm{RI} 200,000-630,000 / \mu \mathrm{L})$. The morphologic alterations included basket cells, reactive lymphocytes and abnormal blast lymphocytes (cytoplasmatic vacuolization, nuclear molding, prominent nucleoli). Abnormalities in the serum biochemical profile (Automated analyzer CM200) $)^{2}$ included mild hyperalbuminemia (36 g/L, RI 21-33 g/L), moderated increment in ALT (350 U/L, RI < $83 \mathrm{U} / \mathrm{L})$ and severe increment in $\operatorname{ALP}(26,366 \mathrm{U} / \mathrm{L}, \mathrm{RI}<$ $93 \mathrm{U} / \mathrm{L})$. Ultrasonography evaluation showed increased liver dimensions. An ELISA point of care test (FIV/FelV SNAP Test) ${ }^{3}$ resulted negative for FIV antibodies but positive for FeLV antigen. Urinalysis revealed moderated bilirubinuria (2+) and proteinuria (30 mg/dL). The bone marrow cytology (Wright stain) revealed high cellularity (85\%, RI < 25\%), increased of M:E ratio (5, RI 1,2-2,2) (Figure 1), rare megakaryocyte lineage, erythroid hypoplasia, myeloid hyperplasia with blast excess (30\%) and dysgranulopoiesis (Figure 2). A positive Sudan Black Stain reaction was positive in granulocytic, monocytic and blast cells. The bone marrow findings suggest acute myeloid leukemia with excess of blasts.

Treatment was initiated with ondansetron (antiemetic), ranitidine (gastric protector), amoxicillin-clavulanic acid (antibiotics), cobamamide and cyproheptadine (orexigens), complex B vitamins and minerals and fluid therapy with Ringer's solution. Additionally, the cat received two blood transfusions within one week. After seven days of treatment, the cat showed slight recovery. The owner declined continuing the treatment and one week after discharge the cat died. Necropsy was declined by the owner.

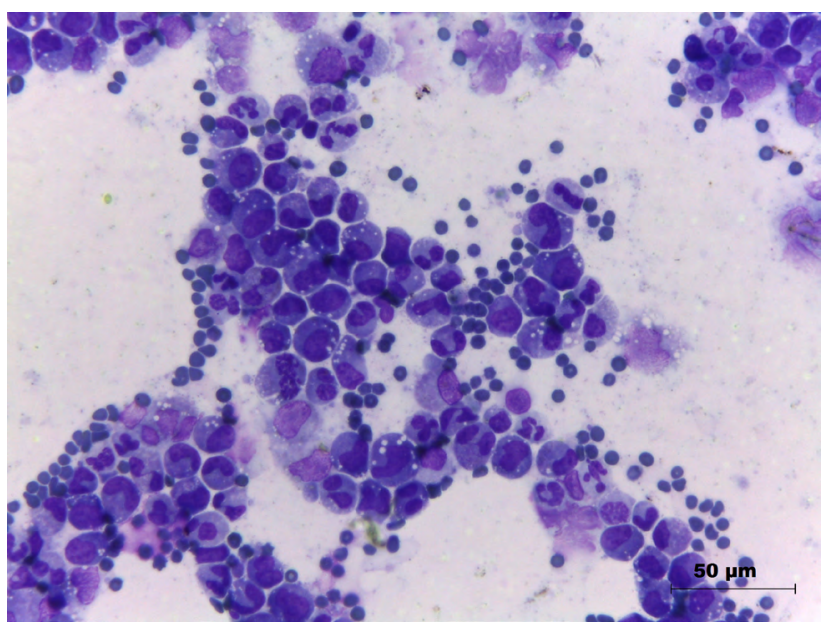

Figure 2. Bone marrow cytology. Myeloid hyperplasia with blast excess and strong maturation asynchrony with pleomorphic blast cells from myeloid lineage. Absence of erythroid precursors. Wright Stain [magnification: 40x]. 


\section{DISCUSSION}

In a FeLV positive cat, cytopenias are common and in this case was well evidenced by the severe non-regenerative anemia and thrombocytopenia, as previously documented [3]. In this case, the anemia may also be attributable to the observed acute myeloid leukemia. Additionally, an increased turnover of the half-life of RBC could be responsible for severe pre-hepatic jaundice, which was clinically evidenced in the cat.

Neutrophilia is an uncommon finding in FeLV-infected cats, even more with the severe degenerative left shift as in the present case. This finding could be associated with the bone marrow cytology, which was evidenced by a myeloid hyperplasia and other abnormalities suggesting myeloid leukemia. Also, lymphopenia is more frequent than lymphocytosis in FeLV-infected cats [4]. In the present case, lymphocytosis was accompanied by a variety of morphological abnormalities in $46 \%$ of the lymphocytes, such as basket cells, reactive lymphocytes, lymphocytic satellitism, cytological vacuolization and anisonucleosis. Although these findings in the peripheral blood could indicate reactive response or neoplastic disease like lymphoid leukemia or lymphoma, the Sudan Black stain of bone marrow cytology demonstrated that most of neoplastic blast cell was from myeloid progenitor lineage.

Hyperalbuminemia in this case could be attributed to the dehydration of the cat. The mild increment in values of serum ALT may have been related to some degree of hepatic origin which was accompanied by enlargement of the organ seen at the ultrasonography exam. However, the most surprising finding in the present case was the excessive value for alkaline phosphatase (ALP) serum activity. ALP has various isoenzymes from liver, bone, gut and kidney origin. Also, an isoenzyme induced by corticosteroids may be present in dogs [5]. The severe increment in ALP in the present case does not have clinical congruency with a hepatic or any other organ lesion; instead it was probably attributed to the neutrophilia. In humans high levels of ALP were observed associated with neutrophilia from damaged or dead neutrophils [4]. It is quite likely that the great amount of blast cells in a bone marrow in the present case may contribute to the high leakage of ALP than in other circumstances. In animals, the leukocyte ALP is found in secondary granules of neutrophils in several species including horses, rabbit and ruminants. This enzyme is absent in canine and feline mature neutrophils while the activity is present in myeloblast and promyelocyte stages [7]. In dogs and cats the high activity of ALP in a bone marrow or others tissues suggest is a specific marker to myelogenous leukemia [8]. Accordingly, the bone marrow and peripheral blood neoplastic proliferation of blast cells from myeloid lineage, could contribute to the high levels of ALP serum activity in this case.

To our best knowledge this is the first report of a FeLV-positive cat exhibiting extremely high serum values of the enzyme ALP, which is apparently attributable to a acute myeloid leukemia.

\section{MANUFACTURERS}

${ }^{1}$ Idexx Laboratories. Westbrook, ME, USA.

${ }^{2}$ Wiener Lab Group. Rosario, Argentina.

${ }^{3}$ Idexx Brasil. São Paulo, SP, Brazil.

Declaration of interest. The authors report no conflicts of interest. The authors alone are responsible for the content and writing of the paper.

\section{REFERENCES}

1 Gleich S. \& Hartmann K. 2009. Hematology and serum biochemistry of Feline Immunodeficiency Virus-infected and Feline Leukemia Virus-infected cats. Journal of Veterinary Internal Medicine. 23: 552-558.

2 Hartmann K. 2012. Clinical aspects of feline retroviruses: a review. Viruses. 4: 2684-2710.

3 Hofmann-Lehmann R., Holznagel E., Ossent P. \& Lutz H. 1997. Parameters of disease progression in long-term experimental feline retrovirus (feline immunodeficiency virus and feline leukemia virus) infections: hematology, clinical chemistry, and lymphocyte subsets. Clinical and Diagnostic Laboratory Immunology. 4: 33-42.

4 Izumi M., Ishikawa J., Takeshita A. \& Maekawa M. 2005. Increased serum Alkaline Phosphatase activity originating from neutrophilic leukocytes. Clinical Chemistry. 51: 1751-1752.

5 Kramer J.W. \& Hoffmann W.E. 1997. Clinical enzymology. In: Kaneko J.J., Harvey J.W. \& Bruss M.L. (Eds). Clinical biochemistry of domestic animals. 5th edn. San Diego: Academic Press, pp.303-325. 
6 Linenberger M.L., Beebe A.M., Pedersen N.C., Abkowitz J.L. \& Dandekar S. 1995. Marrow accessory cell infection and alterations in hematopoiesis accompany severe neutropenia during experimental acute infection with feline immunodeficiency virus. Blood. 85: 941-951.

7 Nabity M.B. \& Ramaiah S.K. 2012. Neutrophil structure and biochemistry. In: Weiss D.J. \& Wardrop K.J. (Eds). Schalm's Veterinary Hematology. 6th edn. Ames: Blackwell Publishing, pp.263-267.

8 Raskin R.E. 2012. Cytochemical Staining. In: Weiss D.J. \& Wardrop K.J. (Eds). Schalm's Veterinary Hematology. 6th edn. Ames: Blackwell Publishing, pp.1141-1156. 